\title{
UNITARY INVERTIBLE GRAPHS OF FINITE RINGS
}

\author{
Tekuri Chalapathi \\ Department of Mathematics \\ Sree Vidyanikethan Engineering College \\ Tirupati, A.P., India \\ e-mail: chalapathi.tekuri@gmail.com \\ AND \\ Shaik SaJAna \\ Department of Mathematics \\ P.R. Govt. Degree College (A) \\ Kakinada, A.P., India \\ e-mail: ssajana.maths@gmail.com
}

\begin{abstract}
Let $R$ be a finite commutative ring with unity. In this paper, we consider set of additive and mutual additive inverses of group units of $R$ and obtain interrelations between them. In general $\varphi\left(Z_{n}\right)$ is even, however we demonstrate that $\varphi(R)$ is odd for any finite commutative ring with unity of $C h a r(R) \neq 2$. Further, we present unitary invertible graph related with self and mutual additive inverses of group units. At long last, we establish a formula for counting the total number of basic and non-basic triangles in the unitary invertible graph.
\end{abstract}

Keywords: finite commutative rings, additive and mutual additive inverses, Euler-function, unitary invertible graphs, basic and non-basic triangles.

2010 Mathematics Subject Classification: 11G15, 11T30, 05C30.

\section{REFERENCES}

[1] M. Afkhami and F. Khosh-Ahang, Unit graphs of rings of polynomials and power series, Arab. J. Math. 2 (2013) 233-246.

doi:10.1007/s40065-013-0067-0

[2] R. Akhtar, M. Boggess, T. Jackson-Henderson, I. Jimenez, R. Karpman, A. Kinzel and D. Pritikin, On the Unitary Cayley Graph of a Finite Ring, Electron. J. Combin. 16 (2009) 1-13. 
[3] M.R. Alfuraidan and Y.F. Zakariya, Inverse graphs associated with finite groups, Electron. J. Graph Theory and Appl. 5 (2017) 142-154.

doi:10.5614/ejgta.2017.5.1.14

[4] N. Ashrafi, H.R. Maimani, M.R. Pournaki and S. Yassemi, Unit Graphs Associated with Rings, Commun. Algebra 38 (2010) 2851-2871. doi:10.1080/00927870903095574

[5] M. Basic and A. Ilic, Polynomials of Unitary Cayley Graphs, Filomat 29 (2015) 2079-2086. doi:10.2298/FIL1509079B

[6] J.A. Beachy and W.D. Blair, Abstract Algebra, Third Edition (Waveland Press, 2006).

[7] N. Biggs, Algebraic Graph Theory, 2nd Edition (Cambridge India, 2016).

[8] J.A. Bondy and U.S.R. Mutry, Graph Theory with Application (Springer India, 2013).

[9] P. Brrizbeitia and R.E. Gaudici, Counting pure $k$-cycles in sequences of Cayley graphs, Discrete Math. 149 (1996) 11-18.

[10] T. Chalapathi, RVMSS. Kiran Kumar and F. Smarandache, Neutrosophic Invertible Graphs of Neutrosophic Rings, New Trends in Neutrosophic Theory and Applications 2 (2018) 209-217.

[11] T. Chalapathi and RVMSS. Kiran Kumar, Self Additive Inverse Elements of Neutrosophic Rings and Fields, Ann. Pure Appl. Math. 13 (2017) 63-72. doi:10.22457/apam.v13n1a7

[12] T. Chalapathi, S. Sajana and D. Bharathi, Classical pairs in $Z_{n}$, Notes on Number Theory and Discrete Math. 26 (2020) 59-69. doi:10.7546/nntdm.2020.26.1.59-69

[13] I. Dejter and R.E. Giudici, On unitary Cayley graphs, J. Combin. Math. Combin. Comput 18 (1995) 121-124.

[14] G. Dresden and W.M. Dymacek, Finding Factors and Factor Rings over the Gaussian Integers, The Mathematical Association of America 112 (2005) 602-611. doi:10.1080/00029890.2005.11920231

[15] S. Huadong, A study of unit graphs and unitary cayley graphs associated with rings, Doctoral Ph.D. Thesis (Memorial University of Newfoundland, 2015).

[16] D.L. James, Applications of Unitary Symmetry and Combinatorics (World Scientific Publishers, 2011).

[17] W.F. Joe and L.S. Robert, Rings Generated by Their Units, J. Algebra 42 (1976) $363-368$.

[18] R. Joy and K. Patra, Some aspects of Unitary addition Cayley graph of Gaussian integers modulo n, Matematika 32 (2016) 43-52.

doi:10.11113/matematika.v32.n1.782 
[19] D. Kiani and M.M.H. Aghaei, On the Unitary Cayley Graphs of a Ring, Electron. J. Combin. 19 (2012) 1-10. doi:doi.org/10.37236/2214

[20] W. Klotz and T. Sander, Some properties of unitary Cayley graphs, Electron. J. Combin. 14 (2007) 1-12. doi: $10.37236 / 963$

[21] X. Liu and S. Zhou, Spectral Properties of Unitary Cayley Graphs of Finite Commutative Rings, Electron. J. Combin. 19 (2012) 1-13.

[22] H.R. Maimani, M.R. Pournaki and S. Yassemi, Rings which are generated by their units: a graph theoretical approach, Elem. Math. 65 (2010) 17-25. doi:10.4171/EM/134

[23] R.G. Raphael, Rings which are generated by their units, J. Algebra 28 (1974) 199-205.

[24] I. Shavitt, Graph Theoretical Concepts for the Unitary Group Approach to the ManyElectron Correlation Problem, International Journal of Quantum Chemistry: Quantum Chemistry Symposium 11 (1977) 131-148. doi:10.1002/qua.560120819

[25] S.G. Telang, Number Theory (Tmh Publisher, 1996).

[26] A. Tripi, Cayley Graphs of Groups and Their Applications, Doctoral Ph.D. Thesis (Missouri State University, 2017).

[27] I.V. Vitaly, Introduction to Graph Theory (Nova Science Publishers. Inc. New York, 2009).

Received 11 April 2020

Revised 2 July 2020

Accepted 31 January 2021 